\title{
Prevalência de discriminação percebida por orientação sexual nos serviços de saúde do Brasil: Pesquisa Nacional de Saúde, 2013
}

\section{Prevalence of discrimination perceived by sexual orientation in Brazilian health services: National Health Survey, 2013}

\author{
Rafael Tavares Jomar ${ }^{1}$ (D), Vitor Augusto de Oliveira Fonseca² (D), \\ Dandara de Oliveira Ramos ${ }^{3}$ (D), Gerson Luiz Marinho ${ }^{4}$ (D), Raphael Mendonça Guimarães ${ }^{5}$ (D), \\ Margareth Cristina de Almeida Gomes ${ }^{6}$ (D), Karina Cardoso Meira 7 (D) \\ 'Instituto Nacional de Câncer José Alencar Gomes da Silva, Coordenação de Assistência - Rio de Janeiro (RJ), Brasil. \\ ${ }^{2}$ Serviço Social do Comércio, Departamento Nacional- Rio de Janeiro (RJ), Brasil. \\ ${ }^{3}$ Universidade Federal da Bahia, Instituto de Saúde Coletiva - Salvador (BA), Brasil. \\ ${ }^{4}$ Universidade Federal do Rio de Janeiro, Escola de Enfermagem Anna Nery - Rio de Janeiro (RJ), Brasil. \\ ${ }^{5}$ Fundação Oswaldo Cruz, Escola Politécnica de Saúde Joaquim Venâncio - Rio de Janeiro (RJ), Brasil. \\ ${ }^{6}$ Universidade Federal do Rio de Janeiro, Escola de Enfermagem Anna Nery - Rio de Janeiro (RJ), Brasil. \\ ?Universidade Federal do Rio Grande do Norte, Escola de Saúde - Natal (RN), Brasil.
}

\begin{abstract}
Como citar: Jomar RT, Fonseca VAO, Ramos DO, Marinho GL, Guimarães RM, Gomes MCA, et al. Prevalência de discriminação percebida por orientação sexual nos serviços de saúde do Brasil: Pesquisa Nacional de Saúde, 2013. Cad Saúde Colet, 2021;29(esp.):187-198. https://doi.org/10.1590/1414-462X202199010416
\end{abstract}

\section{Resumo}

Introdução: A associação entre discriminação por orientação sexual e desfechos negativos em saúde é cada vez mais estabelecida na literatura. Entretanto, poucos estudos exploram a discriminação por orientação sexual nos serviços de saúde em amostras representativas da população geral. Objetivo: Estimar a prevalência de discriminação percebida por orientação sexual nos serviços de saúde do Brasil. Método: Este estudo transversal utilizou dados da Pesquisa Nacional de Saúde 2013. A prevalência de discriminação percebida por orientação sexual nos serviços de saúde foi estimada de forma global, e sua associação com características sociodemográficas e de uso de serviços de saúde foi investigada por meio de regressão de Poisson com variância robusta que calculou razões de prevalências. Resultados: A prevalência global do fenômeno sob investigação foi igual a 0,18\%. Embora estatisticamente significativa, a magnitude das associações observadas foi tão pequena, que nenhuma das características sociodemográficas e de uso de serviços de saúde investigadas pareceu ser capaz de, pragmaticamente, influenciar o risco de uma pessoa sofrer discriminação por orientação sexual nos serviços de saúde do Brasil. Conclusão: $O$ estudo reforça a importância de instaurar ações de promoção do respeito à diversidade sexual nos serviços de saúde do Brasil. Palavras-chave: discriminação sexual e de gênero; minorias sexuais e de gênero; serviços de saúde; inquéritos epidemiológicos; Brasil.

\begin{abstract}
Background: The association between discrimination based on sexual orientation and adverse health outcomes is increasingly observed in the literature. However, few studies explore discrimination based on sexual orientation in health services in representative samples of the overall population. Objective: To estimate the prevalence of discrimination perceived by sexual orientation in Brazilian health servicesl. Method: This cross-sectional study used data from the 2013 National Health Survey. The prevalence of discrimination perceived by sexual orientation in health services was globally estimated and its association
\end{abstract}

Este é um artigo publicado em acesso aberto (Open Access) sob a licença Creative Commons Attribution, que permite uso, distribuição e reprodução em qualquer meio, sem restrições desde que o trabalho original seja corretamente citado.

\footnotetext{
Trabalho realizado a partir de dados secundários, sem pesquisa de campo. Correspondência: Rafael Tavares Jomar. E-mail: rafaeljomar@yahoo.com.br Fonte de financiamento: nenhuma.

Conflito de interesses: nada a declarar.

Recebido em: Ago. 11, 2021. Aprovado em: Out. 09, 2021
} 
with sociodemographic characteristics and those regarding health services was investigated using Poisson regression producing robust variance that calculated prevalence ratios. Results: The global prevalence of the phenomenon investigated was equal to $0.18 \%$. Although statistically significant, the magnitude of the observed associations was so small that none of the sociodemographic characteristics and the use of health services investigated seemed to be able to pragmatically influence the risk of a person being discrimined due to his/her sexual orientation in Brazilian health servicesl. Conclusion: The study stresses the importance of establishing actions to promote respect for sexual diversity in Brazilian health services.

Keywords: sexual and gender discrimination; sexual and gender minorities; health services; health surveys; Brazil.

\section{INTRODUÇÃO}

A Carta dos Direitos dos Usuários da Saúde assegura ao cidadão brasileiro acesso integral ao sistema de saúde do país, seja ele público, seja ele privado, e um de seus princípios garante que todo cidadão tem direito ao atendimento humanizado, acolhedor e livre de qualquer discriminação'. Ainda assim, integrantes das minorias sexuais, especialmente os que se identificam como lésbicas, gays, bissexuais, travestis, transexuais ou transgêneros (LGBT), são vulneráveis à discriminação nos espaços e no atendimento dos serviços de saúde do Brasil $^{2}$, isso porque, historicamente, predomina no país um sistema social heteronormativo que compreende a homossexualidade como uma categoria de orientação sexual desviante ${ }^{3}$.

A discriminação por orientação sexual tem sido pouco explorada no âmbito da saúde coletiva no Brasil, sendo mais pronunciada a escassez de estudos sobre esse fenômeno nos serviços de saúde em amostras representativas da população. Um estudo realizado com 4.176 homens que fazem sexo com homens em 12 capitais brasileiras em 2016 constatou que $9 \%$ deles foram mal atendidos ou maltratados em serviços de saúde ou por profissionais de saúde nos últimos 12 meses antes da pesquisa por conta de sua orientação sexual ${ }^{4}$. Ao explorar a relação entre a adoção de cuidados com saúde e as representações relativas ao gênero, sexualidade e corpo, um estudo qualitativo realizado na Grande São Paulo com mulheres que fazem sexo com mulheres descreveu, além de situações discriminatórias em serviços de saúde, maior dificuldade em acessar consulta ginecológica e exame Papanicolau entre aquelas que nunca tiveram sexo com homens ou que possuíam uma gramática corporal masculinizada ${ }^{5}$. No que diz respeito a travestis e transexuais, estudos de revisão apontam que essas pessoas têm dificuldade de acesso universal, integral e equânime aos serviços de saúde do Brasil, com relatos de experiências discriminatórias e problemas na comunicação com profissionais de saúde, gerando exclusão e marginalização ${ }^{6,7}$.

À vista disso, a discriminação por orientação sexual pode ser considerada um problema de saúde pública ${ }^{8}$ no Brasil também. De mais a mais, a literatura tem evidenciado uma consistente associação entre discriminação por orientação sexual e desfechos negativos em saúde entre pessoas LGBT, tais como uso de substâncias psicoativas ${ }^{9,10}$, uso abusivo de álcool ${ }^{11}$, sintomas de estresse psicológico ${ }^{12}$ e de depressão ${ }^{13,14}$, ideação suicida ${ }^{15}$, comportamentos sexuais de risco ${ }^{16,17}$ e histórico de infecções sexualmente transmissíveis ${ }^{18}$.

Embora a associação entre discriminação por orientação sexual e desfechos negativos em saúde esteja cada vez mais estabelecida na literatura, ainda são escassos estudos sobre o fenômeno discriminação por orientação sexual no âmbito dos serviços de saúde em amostras representativas da população geral, estudos esses que são fundamentais para orientar políticas de saúde públicå . Considerando que a discriminação por orientação sexual é um importante determinante social da saúde da população $\mathrm{LGBT}^{19}$ e visando contribuir para o avanço do conhecimento sobre o tema e evidenciar particularidades do país, o objetivo deste estudo foi estimar a prevalência de discriminação percebida por orientação sexual nos serviços de saúde do Brasil.

\section{MÉTODO}

Este estudo transversal utilizou dados da Pesquisa Nacional de Saúde (PNS), um inquérito de base domiciliar conduzido em 2013 pelo Ministério do Planejamento, Orçamento e Gestão, em convênio com o Ministério da Saúde, e operacionalizado pelo Instituto Brasileiro de Geografia e Estatística (IBGE). O principal objetivo da PNS foi avaliar a saúde, os modos de 
vida e a atenção à saúde da população adulta brasileira ${ }^{20}$, e seu plano amostral complexo utilizou conglomerados em três estágios: os setores censitários foram as unidades primárias; os domicílios, as secundárias; e os moradores com 18 anos de idade ou mais, as terciárias. Os setores censitários foram estratificados de acordo com quatro critérios: administrativo (subdividiu as unidades da federação em capital, restante da região metropolitana e demais municípios), geográfico (subdividiu as capitais e outros municípios de grande porte em mais estratos: distrito, subdistrito e bairros), de situação (subdividiu os estratos anteriores em rural e urbano) e estatístico. Foram visitados 69.954 domicílios ocupados, nos quais foi selecionado morador para entrevista individual, dos quais 60.202 aceitaram participar da PNS (taxa de resposta de $86 \%)^{21}$.

A coleta de dados foi realizada por entrevistadores devidamente treinados utilizando-se de computadores de mão. Para o adulto selecionado no domicílio, foi aplicado questionário sobre características sociodemográficas, autoavaliação do estado de saúde, estilo de vida, morbidades físicas e mentais, acidentes e violências, saúde da mulher e de crianças menores de 2 anos de idade, saúde bucal, saúde do idoso e desempenho do sistema de saúde. Além disso, foram realizadas aferições de medidas antropométricas, pressão arterial e coleta de material biológico. Informações adicionais sobre aspectos metodológicos da PNS estão detalhadamente apresentadas em outra publicação ${ }^{21}$.

A discriminação percebida por orientação sexual nos serviços de saúde foi aferida por meio da seguinte questão: “O(a) sr(a) já se sentiu discriminado(a) ou tratado(a) pior do que as outras pessoas no serviço de saúde, por algum médico ou outro profissional de saúde por motivo de preferência sexual?". E as opções de resposta eram "sim" ou "não".

A prevalência de discriminação percebida por orientação sexual nos serviços de saúde e o respectivo intervalo de confiança de $95 \%$ (IC95\%) foram estimados de forma global e segundo categorias de variáveis sociodemográficas e de uso de serviços de saúde, quais sejam: região de residência (Norte, Nordeste, Centro-Oeste, Sudeste e Sul), idade (em faixas etárias: 18-39, 40-59 e $\geq 60$ anos) escolaridade (sem instrução ou fundamental incompleto, fundamental completo ou médio incompleto, médio completo ou superior incompleto e superior completo), posse de plano privado de saúde (sim e não), última vez que consultou um médico (há menos de um mês e há mais de um mês) e última consulta médica feita no Sistema Único de Saúde (sim e não). Conforme as categorias de variáveis sociodemográficas e de uso de serviços de saúde, foram calculadas razões de prevalências (RP) brutas e os respectivos IC95\% por meio de regressão de Poisson com variância robusta. Em seguida, modelos de regressão de Poisson com variância robusta estimaram as RP ajustadas ao incluírem todas as variáveis que na etapa anterior apresentaram, em pelo menos uma categoria, IC95\% estatisticamente significativo, isto é, não continham o valor 1,00.

Em todas as etapas de análise de dados, conduzidas no programa Stata, versão 15.0, foram utilizados procedimentos que consideraram o desenho complexo da amostra da PNS. Tanto a prevalência quanto as RP foram estimadas separadamente para as pessoas do sexo masculino de cor/raça branca e não branca e para as pessoas do sexo feminino de cor/raça branca e não branca, ou seja, seus cálculos foram estratificados por sexo e cor/raça. A condução da análise de dados estratificada por essas variáveis está baseada no pressuposto de que o gênero e a cor/raça são estruturantes na sociedade brasileira, interferindo nos desfechos de saúde, doença e morte ${ }^{22}$.

A PNS foi aprovada pela Comissão Nacional de Ética em Pesquisa em 26 de junho de 2013 (Parecer no 328.159), e todos os entrevistados assinaram o Termo de Consentimento Livre e Esclarecido.

\section{RESULTADOS}

A Tabela 1 apresenta as características sociodemográficas e de uso de serviços de saúde das pessoas que já se sentiram discriminadas por motivo de orientação sexual nos serviços de saúde do Brasil. A prevalência global do fenômeno sob investigação foi igual a 0,18\%, sendo que entre as pessoas do sexo masculino brancas e não brancas as prevalências foram, respectivamente, de $0,33 \%$ e $0,14 \%$ e entre as pessoas do sexo feminino brancas e não brancas as prevalências foram de $0,13 \%$ e $0,18 \%$, respectivamente (Tabela 2 ). 
Tabela 1. Características das pessoas que já se sentiram discriminadas por motivo de orientação sexual nos serviços de saúde. Pesquisa Nacional de Saúde, Brasil, 2013

\begin{tabular}{|c|c|c|}
\hline Variáveis/categorias & $\mathbf{n}$ & $\%$ \\
\hline Total & 153 & 100,00 \\
\hline \multicolumn{3}{|l|}{ Região } \\
\hline Norte & 51 & 16,34 \\
\hline Nordeste & 38 & 32,09 \\
\hline Centro-Oeste & 28 & 13,48 \\
\hline Sudeste & 21 & 31,34 \\
\hline Sul & 15 & 6,75 \\
\hline \multicolumn{3}{|l|}{ Sexo } \\
\hline Masculino & 72 & 53,69 \\
\hline Feminino & 81 & 46,31 \\
\hline \multicolumn{3}{|l|}{ Cor/raça } \\
\hline Branca & 60 & 46,27 \\
\hline Não branca* & 93 & 53,73 \\
\hline \multicolumn{3}{|l|}{ Faixa etária } \\
\hline 18-39 anos & 97 & 60,90 \\
\hline 40-59 anos & 43 & 28,68 \\
\hline$\geq 60$ anos & 13 & 10,42 \\
\hline \multicolumn{3}{|l|}{ Escolaridade ${ }^{* *}$} \\
\hline Sem instrução ou fundamental incompleto & 44 & 26,28 \\
\hline Fundamental completo ou médio incompleto & 30 & 15,96 \\
\hline Médio completo ou superior incompleto & 55 & 40,12 \\
\hline Superior completo & 21 & 17,64 \\
\hline \multicolumn{3}{|l|}{ Posse de plano privado de saúde } \\
\hline Sim & 27 & 21,68 \\
\hline Não & 126 & 78,32 \\
\hline \multicolumn{3}{|l|}{ Última vez que consultou um médico } \\
\hline Há menos de um mês & 55 & 45,26 \\
\hline Há mais de um mês & 98 & 54,74 \\
\hline \multicolumn{3}{|l|}{ Última consulta médica feita no SUS** } \\
\hline Sim & 66 & 45,52 \\
\hline Não & 43 & 54,48 \\
\hline
\end{tabular}

*Categoria composta por pretos $(n=20 ; 10,91 \%)$, pardos $(n=70 ; 42,05 \%)$, amarelos $(n=1 ; 0,56 \%)$ e indígenas $(n=2 ; 0,21 \%)$;

**Excluídas do cálculo percentual as informações faltantes. 
Tabela 2. Prevalência e intervalo de confiança de $95 \%$ de discriminação percebida por orientação sexual nos serviços de saúde conforme sexo e cor/raça. Pesquisa Nacional de Saúde, Brasil, 2013

\begin{tabular}{|c|c|c|c|c|}
\hline \multirow{2}{*}{ Variáveis/categorias } & \multicolumn{2}{|c|}{ Masculino } & \multicolumn{2}{|c|}{ Feminino } \\
\hline & Branca & Não branca & Branca & Não branca \\
\hline Global & $0,33(0,19-0,57)$ & $0,14(0,09-0,21)$ & $0,13(0,08-0,21)$ & $0,18(0,11-0,29)$ \\
\hline \multicolumn{5}{|l|}{ Região } \\
\hline Norte & $1,76(0,05-5,16)$ & $0,39(0,19-0,81)$ & $0,10(0,03-0,28)$ & $0,26(0,15-0,44)$ \\
\hline Nordeste & $0,29(0,12-0,67)$ & $0,21(0,10-0,42)$ & $0,11(0,04-0,28)$ & $0,24(0,11-0,52)$ \\
\hline Centro-Oeste & $0,04(0,01-0,19)$ & $0,39(0,16-0,93)$ & $0,43(0,19-0,96)$ & $0,37(0,13-1,07)$ \\
\hline Sudeste & $0,26(0,13-0,52)$ & $0,03(0,01-0,13)$ & $0,11(0,04-0,29)$ & $0,10(0,03-0,34)$ \\
\hline Sul & $0,01(0,01-0,17)$ & $0,10(0,03-0,35)$ & $0,08(0,03-0,24)$ & $0,17(0,05-0,57)$ \\
\hline \multicolumn{5}{|l|}{ Faixa etária } \\
\hline $18-39$ anos & $0,48(0,24-0,96)$ & $0,20(0,12-0,32)$ & $0,18(0,10-0,32)$ & $0,20(0,10-0,38)$ \\
\hline 40-59 anos & $0,15(0,05-0,39)$ & $0,12(0,05-0,27)$ & $0,13(0,05-0,33)$ & $0,20(0,09-0,44)$ \\
\hline$\geq 60$ anos & $0,29(0,10-0,81)$ & $0,02(0,01-0,12)$ & $0,01(0,01-0,02)$ & $0,09(0,02-0,45)$ \\
\hline \multicolumn{5}{|l|}{ Escolaridade } \\
\hline Sem instrução ou fundamental incompleto & $0,09(0,02-0,37)$ & $0,08(0,04-0,19)$ & $0,12(0,04-0,32)$ & $0,15(0,06-0,34)$ \\
\hline Fundamental completo ou médio incompleto & $0,10(0,03-0,35)$ & $0,10(0,03-0,29)$ & $0,32(0,14-0,74)$ & $0,16(0,05-0,49)$ \\
\hline Médio completo ou superior incompleto & $0,62(0,29-1,30)$ & $0,18(0,09-0,36)$ & $0,08(0,02-0,23)$ & $0,19(0,09-0,42)$ \\
\hline Superior completo & $0,36(0,13-0,98)$ & $0,46(0,20-1,04)$ & $0,11(0,04-0,28)$ & $0,32(0,08-1,20)$ \\
\hline \multicolumn{5}{|l|}{ Posse de plano privado de saúde } \\
\hline Sim & $0,24(0,11-0,52)$ & $0,11(0,04-0,29)$ & $0,06(0,01-0,21)$ & $0,15(0,05-0,47)$ \\
\hline Não & $0,38(0,19-0,75)$ & $0,15(0,10-0,24)$ & $0,17(0,10-0,29)$ & $0,19(0,11-0,32)$ \\
\hline \multicolumn{5}{|l|}{ Última vez que consultou um médico } \\
\hline Há menos de um mês & $0,39(0,17-0,88)$ & $0,33(0,18-0,59)$ & $0,10(0,04-0,23)$ & $0,33(0,17-0,62)$ \\
\hline Há mais de um mês & $0,32(0,16-0,62)$ & $0,08(0,05-0,15)$ & $0,15(0,08-0,26)$ & $0,09(0,05-0,17)$ \\
\hline \multicolumn{5}{|l|}{ Última consulta médica feita no SUS } \\
\hline Sim & $0,17(0,05-0,50)$ & $0,15(0,08-0,29)$ & $0,16(0,08-0,31)$ & $0,19(0,10-0,36)$ \\
\hline Não & $1,13(0,87-1,47)$ & $0,20(0,09-0,43)$ & $0,09(0,04-0,23)$ & $0,18(0,07-0,46)$ \\
\hline
\end{tabular}

Para o sexo masculino, os modelos de regressão multivariada mostraram que a prevalência de discriminação percebida por orientação sexual nos serviços de saúde do Brasil foi $1 \%$ maior entre as pessoas de cor/raça não branca com idade $\geq 60$ anos consultadas por um médico há mais de um mês e $1 \%$ menor entre os residentes das regiões Norte, Nordeste e Centro-Oeste. Em contrapartida, entre aquelas de cor/raça branca a prevalência foi menor, da ordem de $3 \% \mathrm{e}$ $1 \%$, entre os residentes da região Norte e com ensino médio completo ou superior incompleto, respectivamente (Tabela 3 ). 
Tabela 3. Razão de prevalência e intervalo de confiança de $95 \%$ de discriminação percebida por orientação sexual nos serviços de saúde entre pessoas do sexo masculino conforme cor/raça. Pesquisa Nacional de Saúde, Brasil, 2013

\begin{tabular}{|c|c|c|c|c|}
\hline \multirow{2}{*}{ Variáveis/categorias } & \multicolumn{2}{|c|}{ Razão de prevalência bruta } & \multicolumn{2}{|c|}{ Razão de prevalência ajustada } \\
\hline & Branca & Não branca & Branca & Não branca \\
\hline \multicolumn{5}{|l|}{ Região } \\
\hline Norte & $0,99(0,98-1,01)$ & $0,99(0,99-0,99)$ & $0,97(0,96-0,97)$ & $0,99(0,99-0,99)$ \\
\hline Nordeste & $0,99(0,99-1,01)$ & $0,99(0,99-0,99)$ & $0,99(0,99-1,01)$ & $0,99(0,99-0,99)$ \\
\hline Centro-Oeste & $1,01(1,01-1,01)$ & $0,99(0,99-0,99)$ & $1,01(0,99-1,01)$ & $0,99(0,99-0,99)$ \\
\hline Sudeste & 1,00 & 1,00 & 1,00 & 1,00 \\
\hline Sul & $1,01(1,01-1,01)$ & $0,99(0,99-1,01)$ & $1,01(0,99-1,01)$ & $0,99(0,99-1,01)$ \\
\hline \multicolumn{5}{|l|}{ Faixa etária } \\
\hline 18-39 anos & 1,00 & 1,00 & - & 1,00 \\
\hline 40-59 anos & $1,01(0,99-1,01)$ & $1,01(0,99-1,01)$ & - & $1,01(0,99-1,01)$ \\
\hline$\geq 60$ anos & $1,01(0,99-1,01)$ & $1,01(1,01-1,01)$ & - & $1,01(1,01-1,01)$ \\
\hline \multicolumn{5}{|l|}{ Escolaridade } \\
\hline Sem instrução ou fundamental incompleto & 1,00 & 1,00 & 1,00 & - \\
\hline Fundamental completo ou médio incompleto & $0,99(0,99-1,01)$ & $0,99(0,99-1,01)$ & $0,99(0,99-1,01)$ & - \\
\hline Médio completo ou superior incompleto & $0,99(0,99-0,99)$ & $0,99(0,99-1,01)$ & $0,99(0,99-0,99)$ & - \\
\hline Superior completo & $0,99(0,99-1,01)$ & $0,99(0,99-1,01)$ & $0,99(0,99-1,01)$ & - \\
\hline \multicolumn{5}{|l|}{ Posse de plano privado de saúde } \\
\hline Sim & 1,00 & 1,00 & - & - \\
\hline Não & $0,99(0,99-1,01)$ & $0,99(0,99-1,01)$ & - & - \\
\hline \multicolumn{5}{|l|}{ Última vez que consultou um médico } \\
\hline Há menos de um mês & 1,00 & 1,00 & - & 1,00 \\
\hline Há mais de um mês & $1,01(0,99-1,01)$ & $1,01(1,01-1,01)$ & - & $1,01(1,01-1,01)$ \\
\hline \multicolumn{5}{|l|}{ Última consulta médica feita no SUS } \\
\hline Sim & 1,00 & 1,00 & 1,00 & - \\
\hline Não & $0,99(0,99-0,99)$ & $0,99(0,99-1,01)$ & $0,99(0,99-0,99)$ & - \\
\hline
\end{tabular}

Para o sexo feminino, a prevalência de discriminação percebida por orientação sexual nos serviços de saúde do Brasil foi $1 \%$ maior entre as pessoas de cor/raça não branca consultadas por um médico há mais de um mês e entre aquelas de cor/raça branca com idade $\geq 60$ anos. Dado que, tanto no grupo de cor/raça branca quanto no grupo de cor/raça não branca, somente uma variável mostrou IC95\% estatisticamente significativo, modelos de regressão multivariada para o sexo feminino não foram testados (Tabela 4). 
Tabela 4. Razão de prevalência e intervalo de confiança de $95 \%$ de discriminação percebida por orientação sexual nos serviços de saúde entre pessoas do sexo feminino conforme cor/raça. Pesquisa Nacional de Saúde, Brasil, 2013

\begin{tabular}{|c|c|c|}
\hline \multirow{2}{*}{ Variáveis/categorias } & \multicolumn{2}{|c|}{ Razão de prevalência bruta } \\
\hline & Branca & Não branca \\
\hline \multicolumn{3}{|l|}{ Região } \\
\hline Norte & $1,01(0,99-1,01)$ & $0,99(0,99-1,01)$ \\
\hline Nordeste & $0,99(0,99-1,01)$ & $0,99(0,99-1,01)$ \\
\hline Centro-Oeste & $0,99(0,99-1,01)$ & $0,99(0,99-1,01)$ \\
\hline Sudeste & 1,00 & 1,00 \\
\hline Sul & $1,01(0,99-1,01)$ & $0,99(0,99-1,01)$ \\
\hline \multicolumn{3}{|l|}{ Faixa etária } \\
\hline 18-39 anos & 1,00 & 1,00 \\
\hline $40-59$ anos & $1,01(0,99-1,01)$ & $0,99(0,99-1,01)$ \\
\hline$\geq 60$ anos & $1,01(1,01-1,01)$ & $1,01(0,99-1,01)$ \\
\hline \multicolumn{3}{|l|}{ Escolaridade } \\
\hline Sem instrução ou fundamental incompleto & 1,00 & 1,00 \\
\hline Fundamental completo ou médio incompleto & $0,99(0,99-1,01)$ & $0,99(0,99-1,01)$ \\
\hline Médio completo ou superior incompleto & $1,01(0,99-1,01)$ & $0,99(0,99-1,01)$ \\
\hline Superior completo & $1,01(0,99-1,01)$ & $0,99(0,99-1,01)$ \\
\hline \multicolumn{3}{|l|}{ Posse de plano privado de saúde } \\
\hline Sim & 1,00 & 1,00 \\
\hline Não & $0,99(0,99-1,01)$ & $0,99(0,99-1,01)$ \\
\hline \multicolumn{3}{|l|}{ Última vez que consultou um médico } \\
\hline Há menos de um mês & 1,00 & 1,00 \\
\hline Há mais de um mês & $0,99(0,99-1,01)$ & $1,01(1,01-1,01)$ \\
\hline \multicolumn{3}{|l|}{ Última consulta médica feita no SUS } \\
\hline Sim & 1,00 & 1,00 \\
\hline Não & $1,01(0,99-1,01)$ & $1,01(0,99-1,01)$ \\
\hline
\end{tabular}

\section{DISCUSSÃO}

Embora a prevalência de discriminação percebida por orientação sexual nos serviços de saúde do Brasil pareça baixa, vale ressaltar que o estudo foi realizado com amostra representativa da população adulta brasileira, e não com a população do país que se identifica como LGBT. Porém, como a discriminação por orientação sexual contra heterossexuais é pouco provável, uma vez que a heteronormatividade presume a heterossexualidade como a orientação sexual padrão, o que pode resultar, portanto, em discriminação contra pessoas $L G B T^{23}$, é razoável supor que as pessoas que afirmaram já se sentiram discriminadas por motivo de orientação sexual nos serviços de saúde sejam integrantes das minorias sexuais. Mesmo diante disso, a prevalência encontrada pelo estudo deve ser cautelosamente apreciada, já que informações sobre orientação sexual e identidade de gênero não foram coletadas pela PNS. Entretanto, caso tais informações tivessem sido coletadas e aqui avaliadas, acredita-se que uma prevalência 
maior do fenômeno sob escrutínio poderia ter sido encontrada entre pessoas LGBT, pois uma recente revisão sistemática que investigou experiências de discriminação de integrantes das minorias sexuais em serviços de saúde descreveu frequências que variaram de $2 \%$ a $41,8 \%{ }^{24}$.

Um inquérito conduzido na China, país que abriga a maior comunidade LGBT do mundo em valores absolutos, avaliou a discriminação percebida por orientação sexual em diversos ambientes entre integrantes das minorias sexuais de todo o país e apontou que, em serviços de saúde, as experiências de discriminação entre transgêneros eram mais frequentes que entre lésbicas, gays e bissexuais ${ }^{25}$. Com base nos dados do National Transgender Discrimination Survey, estudo que avaliou a discriminação em serviços de saúde entre transgêneros nos Estados Unidos, aqueles que eram sempre reconhecidos como transgênero tinham maiores chances de sofrer discriminação nesses ambientes do que aqueles que nunca eram reconhecidos como tal ${ }^{26}$. Os resultados desses estudos refletem, em certa medida, diferenças na discriminação percebida em serviços de saúde entre os integrantes das minorias sexuais, uma vez que essas experiências parecem afetar mais as pessoas transgênero que as outras, corroborando a hipótese de que, entre transgêneros, é a identidade de gênero (e não a orientação sexual) a causa da discriminação²7.

De modo geral, ações discriminatórias acometem mais as pessoas transgênero porque elas possuem vivências e expressões de gênero discordantes daquelas esperadas pela sociedade heteronormativa ${ }^{28}$. No tocante aos fatores que podem contribuir para a discriminação contra transgêneros em serviços de saúde especificamente, cabe destacar que eles são complexos e incluem os sentimentos de incerteza e de falta de competência cultural em relação a essa população por parte dos médicos, as suposições dos profissionais de saúde de que essas pessoas podem ser mentalmente instáveis ou ter problemas psiquiátricos ${ }^{29,30}$ e o estigma decorrente da susceptibilidade delas às infecções sexualmente transmissíveis ${ }^{31}$.

No contexto, acredita-se que diferenças relacionadas ao sexo e à cor/raça não tenham sido observadas no estudo porque talvez seja a expressão de gênero o motivo mais importante para a ocorrência de discriminação contra integrantes de minorias sexuais nos serviços de saúde do Brasil. Expressão de gênero é como a pessoa manifesta publicamente a sua identidade de gênero, como perfomatiza o gênero por meio do corpo, do nome social, da vestimenta, do corte de cabelo, dos comportamentos, da voz ou de características corporais e da forma como interage com outras pessoas, o que nem sempre corresponde ao sexo biológico ${ }^{32}$. Estudos anteriores parecem corroborar esse entendimento, pois mostraram que integrantes de minorias sexuais foram menos expostos à discriminação por orientação sexual em serviços de saúde quando os profissionais presumiam que eles eram heterossexuais $s^{33-35}$.

Embora estatisticamente significativa, a magnitude das associações observadas no estudo é tão pequena, que nenhuma das características sociodemográficas e de uso de serviços de saúde investigadas parece ser capaz de, pragmaticamente, influenciar o risco de uma pessoa LGBT sofrer discriminação nos serviços de saúde do Brasil. Talvez isso se deva ao fato de o sistema social brasileiro ser heteronormativo, tornando, assim, as minorias sexuais vulneráveis à discriminação nos espaços e no atendimento dos serviços de saúde do país ${ }^{2}$ a despeito de outros atributos.

Soma-se a isso a evidência gerada por uma revisão sistemática de literatura que, ao identificar as dificuldades associadas à homossexualidade no acesso aos serviços de saúde e na utilização deles, concluiu que a orientação não heterossexual é um fator determinante nas dificuldades de acesso aos cuidados de saúde em razão da discriminação e do preconceito sofrido pela população $\mathrm{LGBT}^{36}$. Assim, no Brasil, mesmo após vencida a barreira da orientação sexual desviante no acesso a um serviço de saúde, parece não haver determinantes sociodemográficos ou de uso de serviços de saúde capazes de aumentar ou diminuir o risco de uma pessoa LGBT sofrer discriminação nesses espaços.

Convém destacar, portanto, a relevância da Política Nacional de Saúde Integral LGBT², que foi instituída no Brasil em 2011 com o objetivo principal de promover a saúde integral da população LGBT, eliminando a discriminação e o preconceito institucional e contribuindo para a redução das desigualdades e para a consolidação do SUS como sistema universal, integral e equitativo. Logo, essa política deve ser efetivada ao ser considerada no planejamento de ações de capacitação voltadas para profissionais de saúde e de apoio (recepcionistas e seguranças, por exemplo) com o propósito de aperfeiçoar o acolhimento e o atendimento desse grupo 
populacional em serviços de saúde brasileiros. Desse modo, a promoção do respeito à diversidade sexual estaria sendo trabalhado com vistas à desconstrução de estereótipos que apoiam a discriminação contra pessoas que, por se identificarem como LGBT, não se enquadram no padrão heteronormativo imposto social e culturalmente no Brasil.

Evidências de um estudo sueco mostraram que reduções no estigma estrutural contra as minorias sexuais estão associadas a melhores resultados de saúde entre gays e homens que fazem sexo com homens ${ }^{37}$. Embora nos últimos anos o Brasil tenha avançado em importantes pautas concernentes às minorias sexuais, como o reconhecimento jurídico das relações homoafetivas ${ }^{38}$, e, por isso, seja considerado um dos países de renda baixa/média mais inclusivos com pessoas $\mathrm{LGBT}^{8}$, é também o país que mais registra mortes violentas nessa população em todo o mundo: foram 329 casos em 2019, o que corresponde a, aproximadamente, uma morte a cada 26 horas $^{39}$. Os homicídios contra pessoas LGBT no Brasil são, em geral, "crimes de ódio" e um grave problema de saúde pública por vitimizar jovens, principalmente os transgêneros ${ }^{40}$.

Mesmo diante desse cenário, a Advocacia-Geral da União recorreu da decisão do Supremo Tribunal Federal que, um ano antes (2019), havia reconhecido a discriminação por orientação sexual e identidade de gênero como crime, alegando que o Supremo precisava deixar claro quais atos religiosos não poderiam ser considerados crime de homofobia e transfobia ${ }^{41}$. Diante disso, são enfatizadas a importância e a urgência de combater retrocessos nos direitos das pessoas LGBT no Brasil, a fim de que não seja permitido a nenhum cidadão discriminar um outro com base na orientação sexual ou na identidade de gênero, seja em um templo religioso, seja em um serviço de saúde.

Como as vivências de pessoas LGBT são expressões contra-hegemônicas que desestabilizam as normas sociais e a "heterossexualidade compulsória"42, é possível que as iniciativas decorrentes da luta política do Movimento LGBT no Brasil, que levou o Estado a construir políticas públicas, tenham fomentado reações de segmentos mais conservadores da sociedade brasileira ${ }^{43}$, como a mencionada anteriormente, da Advocacia-Geral da União, instituição que assessora direta, imediata e pessoalmente o presidente da República. No Brasil de 2019-2021, mesmo que o poder institucionalizado - representado pelo presidente da República e por seus aliados políticos - simbolize a vitória do conservadorismo firmado em valores morais cristãos, historicamente machistas e homofóbicos, o efeito rebote, de resistência e de defesa às ações afirmativas, materializa-se nas diversas esferas sociais do país ${ }^{44}$.

Este estudo demanda interpretação à luz de suas limitações: (i) o desenho transversal não permite determinar causalidade; (ii) a pergunta que aferiu o fenômeno sob escrutínio utilizando a expressão"preferência sexual" em vez de "orientação sexual" pode ter influenciado a compreensão do entrevistado sobre a propósito da pergunta e, consequentemente, subestimado a prevalência; (iii) o uso de dados secundários impossibilitou a avaliação da influência de importantes variáveis na prevalência do fenômeno, tais como orientação sexual e identidade de gênero, visto que a PNS não coletou essas informações, bem como inviabilizou testar a suposição de que as pessoas que afirmaram já ter se sentido discriminadas por motivo de orientação sexual nos serviços de saúde eram mesmo integrantes das minorias sexuais; (iv) o denominador utilizado no cálculo da prevalência - a população geral, e não aquela que se identifica como LGBT, essa de fato exposta ao risco de discriminação por orientação sexual certamente subestimou a magnitude dessa medida de frequência, impossibilitando, assim, sua generalização para toda a população LGBT do Brasil. Diante disso, vale mencionar que, embora a PNS tenha perguntado se a condição do entrevistado no domicílio era de "cônjuge ou companheiro(a) do mesmo sexo", a utilização dessa quantidade de pessoas como denominador no cálculo da prevalência excluiria todos as outras pessoas LGBT entrevistadas que responderam que sua posição era de "pessoa responsável pelo domicílio" ou de "filho(a) do responsável e do cônjuge", por exemplo; (v) a provável presença de terceiros no momento da entrevista pode ter subestimado a prevalência, uma vez que a privacidade nesse instante não foi exigida pela PNS, e o entrevistado, por constrangimento, pode ter negado a experiência de discriminação por orientação sexual em serviços de saúde perante outrem.

Não obstante suas limitações, é apontado como ponto forte do estudo o seu procedimento de análise de dados, a qual, além de ter sido estratificada por sexo e cor/raça, calculou a RP 
de discriminação por orientação sexual nos serviços de saúde conforme as características sociodemográficas e de uso de serviços de saúde por meio de regressão de Poisson com variância robusta. Portanto, o presente estudo avança com o conhecimento acerca do fenômeno sob escrutínio, pois outro estudo recentemente publicado fez uso dos mesmos dados da PNS, mas analisou exclusivamente características sociodemográficas por meio de regressão logística ${ }^{45}$. Isto posto, vale sublinhar que em estudos transversais os modelos de regressão de Poisson com variância robusta geram estimativas adequadas de RP, independentemente da prevalência de base ${ }^{46}$. Entretanto, diante de desfechos de baixa prevalência - caso do presente estudo -, os modelos de regressão logística superestimam a razão de chances, quando interpretadas como estimativa de RP, fazendo com que o modelo de regressão de Poisson com variância robusta seja melhor alternativa que a regressão logística em estudos transversais ${ }^{47}$.

Além disso, o estudo reforça a PNS como importante fonte de pesquisa em saúde pública, uma vez que ela se mostrou útil tanto para sinalizar a importância de instaurar ações de promoção do respeito à diversidade sexual nos serviços de saúde do Brasil quanto para expor a necessidade de combater iniquidades nesses ambientes ${ }^{48}$, garantindo, dessa forma, um dos princípios do SUS: a igualdade da assistência à saúde, sem preconceitos ou privilégios de qualquer espécie ${ }^{49}$.

Diante da escassez de estudos sobre discriminação por orientação sexual no âmbito dos serviços de saúde em amostras representativas da população geral, é sublinhada a originalidade do presente estudo, que mostrou que esse fenômeno no Brasil parece não ter determinantes sociodemográficos ou de uso de serviços de saúde capazes de, pragmaticamente, aumentar ou diminuir sua prevalência - o que merece ser minuciosamente examinado em estudos futuros. Sendo assim, seus resultados podem orientar políticas públicas a fim de que todo e qualquer cidadão tenha garantido o seu direito a um atendimento humanizado, acolhedor e livre de discriminação por orientação sexual nos sistemas de saúde do Brasil'.

\section{REFERÊNCIAS}

1. Brasil. Ministério da Saúde. Carta dos direitos dos usuários da saúde. 2. ed. Brasília: Ministério da Saúde; 2007.

2. Brasil. Ministério da Saúde. Política Nacional de Saúde Integral de Lésbicas, Gays, Bissexuais, Travestis e Transexuais - LGBT. Brasília: Ministério da Saúde; 2011.

3. Trevisan JS. Devassos no paraíso: a homossexualidade no Brasil, da colônia à atualidade. 4. ed. Rio de Janeiro: Objetiva; 2018.

4. Magno L, Silva LAV, Guimarães MDC, Veras MASM, Deus LFA, Leal AF, et al. Discrimination based on sexual orientation against MSM in Brazil: a latent class analysis. Rev Bras Epidemiol. 2019;22(22 Supl. 1):e190003. http://dx.doi.org/10.1590/1980-549720190003.supl.1. PMid:31576979.

5. Barbosa RM, Facchini R. Acesso a cuidados relativos à saúde sexual entre mulheres que fazem sexo com mulheres em São Paulo, Brasil. Cad Saude Publica. 2009;25(Supl. 2):291-300. http://dx.doi.org/10.1590/ S0102-311X2009001400011.

6. Araújo IA, Tavares ALB, Souza ACC, Rodrigues FJS. Pessoas transexuais e o acesso aos serviços de saúde no Brasil: revisão integrativa. Cadernos ESP Ceará. 2018;12(2):112-27.

7. Nascimento HM, Sousa A Jr, Barros CRS. O atendimento em saúde a travestis e transexuais: revisão sistemática de literatura (2008-2017). REBEH. 2019;1 (4):40-58. http://dx.doi.org/10.31560/25953206.2018.4.9187.

8. Lamontagne E, d'Elbée M, Ross MW, Carroll A, du Plessis A, Loures L. A socioecological measurement of homophobia for all countries and its public health impact. Eur J Public Health. 2018;28(5):967-72. http:// dx.doi.org/10.1093/eurpub/cky023. PMid:29514190.

9. Brubaker MD, Garrett MT, Dew BJ. Examining the relationship between internalized heterosexism and substance abuse among lesbian, gay and bisexual individuals: a critical review. J LGBT Issues Couns. 2009;3(1):62-89. http://dx.doi.org/10.1080/15538600902754494.

10. Lelutiu-Weinberger C, Pachankis JE, Golub SA, Walker JNJ, Bamonte AJ, Parsons JT. Age cohort differences in the effects of gay-related stigma, anxiety and identification with the gay community on sexual risk and substance use. AIDS Behav. 2013;17(1):340-9. http://dx.doi.org/10.1007/s10461-011-0070-4. PMid:22038078.

11. Weber GN. Using to numb the pain: substance use and abuse among lesbian, gay, and bisexual individuals. J Ment Health Couns. 2008;30(1):31-48. http://dx.doi.org/10.17744/mehc.30.1.2585916185422570. 
12. Díaz RM, Ayala G, Bein E, Henne J, Marin BV.The impact of homophobia, poverty, and racism on the mental health of gay and bissexual Latino men: findings from 3 US cities. Am J Public Health. 2001;91(6):927-32. http://dx.doi.org/10.2105/AJPH.91.6.927. PMid:11392936.

13. Lewis RJ, Derlega VJ, Griffin JL, Krowinski AC. Stressors for gay men and lesbians: life stress, gay-related stress, stigma consciousness, and depressive symptoms. J Soc Clin Psychol. 2003;22(6):716-29. http:// dx.doi.org/10.1521/jscp.22.6.716.22932.

14. Hylton E, Wirtz AL, Zelaya CE, Latkin C, Peryshkina A, Mogilnyi V, et al. Sexual identity, stigma, and depression: the role of the "Anti-gay Propaganda Law" in mental health among men who have sex with men in Moscow, Russia. J Urban Health. 2017;94(3):319-29. http://dx.doi.org/10.1007/s11524-017-0133-6. PMid:28243868.

15. Kohlbrenner V, Deuba K, Karki DK, Marrone G. Perceived discrimination is an independent risk factor for suicidal ideation among sexual and gender minorities in Nepal. PLoS One. 2016;11(7):e0159359. http:// dx.doi.org/10.1371/journal.pone.0159359. PMid:27437996.

16. Ha H, Risser JM, Ross MW, Huynh TN, Nguyen HTM. Homosexuality-related stigma and sexual risk behaviors among men who have sex with men in Hanoi, Vietnam. Arch Sex Behav. 2015;44(2):349-56. http://dx.doi. org/10.1007/s10508-014-0450-8. PMid:25617010.

17. Jeffries WL 4th, Marks G, Lauby J, Murrill CS, Millett GA. Homophobia is associated with sexual behavior that increases risk of acquiring and transmitting HIV infection among black men who have sex with men. AIDS Behav. 2013;17(4):1442-53. http://dx.doi.org/10.1007/s10461-012-0189-y. PMid:22569942.

18. Puckett JA, Newcomb ME, Ryan DT, Swann G, Garofalo R, Mustanski B. Internalized homophobia and perceived stigma: a validation study of stigma measures in a sample of young men who have sex with men. Sex Res Social Policy. 2017;14(1):1-16. http://dx.doi.org/10.1007/s13178-016-0258-5. PMid:28824733.

19. Herek GM, Chopp R, Strohl D. Sexual stigma: putting sexual minority health issues in context. In: Meyer $\mathrm{IH}$, Northridge ME, editors. The health of sexual minorities. Boston: Springer; 2007. p. 171-208. http:// dx.doi.org/10.1007/978-0-387-31334-4_8.

20. Szwarcwald CL, Malta DC, Pereira CA, Vieira MLFP, Conde WL, Souza PRB Jr, et al. Pesquisa Nacional de Saúde no Brasil: concepção e metodologia de aplicação. Cien Saude Colet. 2014;19(2):333-42. http:// dx.doi.org/10.1590/1413-81232014192.14072012. PMid:24863810.

21. Souza PRB Jr, Freitas MPS, Antonaci GA, Szwarcwald CL. Sampling design for the National Health Survey, Brazil 2013. Epidemiol Serv Saude. 2015;24(2):207-16.

22. Werneck J. Racismo institucional e saúde da população negra. Saude Soc. 2016;25(3):535-49. http://dx.doi org/10.1590/s0104-129020162610.

23. Utamsingh PD, Richman LS, Martin JL, Lattanner MR, Chaikind JR. Heteronormativity and practitionerpatient interaction. Health Commun. 2016;31(5):566-74. http://dx.doi.org/10.1080/10410236.2014.979 975. PMid:26421354.

24. Ayhan $\mathrm{CHB}$, Bilgin $\mathrm{H}$, Uluman OT, Sukut O, Yilmaz S, Buzlu S. A systematic review of the discrimination against sexual and gender minority in health care settings. Int J Health Serv. 2020;50(1):44-61. http:// dx.doi.org/10.1177/0020731419885093. PMid:31684808.

25. Wang $Y$, Hu Z, Peng K, Rechdan J, Yang Y, Wu L, et al. Mapping out a spectrum of the Chinese public's discrimination toward the LGBT community: results from a national survey. BMC Public Health 2020;20(1):669. http://dx.doi.org/10.1186/s12889-020-08834-y. PMid:32397988.

26. Rodriguez A, Agardh A, Asamoah BO. Self-reported discrimination in health-care settings based on recognizability as transgender: a cross-sectional study among transgender U.S. citizens. Arch Sex Behav. 2018;47(4):973-85. http://dx.doi.org/10.1007/s10508-017-1028-z. PMid:28785919.

27. Aparicio-García ME. Transgender, sexual orientation, and health. In: Sánchez-Lpez MP, Limiñana-Gras RM, editors. The psychology of gender and health. Cambridge: Academic Press; 2017. p. 143-174. http:// dx.doi.org/10.1016/B978-0-12-803864-2.00005-5.

28. Sousa JA, Rocha TMAC, Barros CRS. Prevalência de discriminação na vida, entre travestis, transexuais e transgêneros. Cadernos de Gênero e Diversidade. 2018;4(1):43-65. http://dx.doi.org/10.9771/cgd. v4i1.24974.

29. Lefkowitz ARF, Mannell J. Sexual health service providers' perceptions of transgender youth in England. Health Soc Care Community. 2017;25(3):1237-46. http://dx.doi.org/10.1111/hsc.12426. PMid:28116847.

30. Snelgrove JW, Jasudavisius AM, Rowe BW, Head EM, Bauer GR. "Completely out-at-sea" with "two-gender medicine": a qualitative analysis of physician-side barriers to providing healthcare for transgender patients. BMC Health Serv Res. 2012;12(1):110. http://dx.doi.org/10.1186/1472-6963-12-110. PMid:22559234. 
31. Carvalho M, Carrara S. Em direito a um futuro trans? Contribuição para a história do movimento de travestis e transexuais no Brasil. Sex Salud Soc (Rio J). 2013;14(14):319-35. http://dx.doi.org/10.1590/ S1984-64872013000200015.

32. Gay \& Lesbian Alliance Against Defamation - GLAAD. GLAAD media reference guide. 10th ed. New York: GLAAD; 2016.

33. Mosack KE, Brouwer AM, Petroll AE. Sexual identity, identity disclosure, and healthcare experiences: is there evidence for differential homophobia in primary care practice? Womens Health Issues. 2013;23(6):e341-6. http://dx.doi.org/10.1016/j.whi.2013.07.004. PMid:24183408.

34. Smith EM, Johnson SR, Guenther SM. Healthcare attitudes and experiences during gynecologic care among lesbians and bisexuals. Am J Public Health. 1985;75(9):1085-7. http://dx.doi.org/10.2105/AJPH.75.9.1085. PMid:4025660.

35. Steele LS, Tinmouth JM, Lu A. Regular healthcare use by lesbians: a path analysis of predictive factors. Fam Pract. 2006;23(6):631-6. http://dx.doi.org/10.1093/fampra/cml030. PMid:16799166.

36. Albuquerque GA, Garcia CL, Quirino GS, Alves MJH, Belém JM, Figueiredo FWS, et al. Access to health services by lesbian, gay, bisexual, and transgender persons: systematic literature review. BMC Int Health Hum Rights. 2016;16(2):2. http://dx.doi.org/10.1186/s12914-015-0072-9. PMid:26769484.

37. Hatzenbuehler ML, Bränström R, Pachankis JE. Societal-level explanations for reductions in sexual orientation mental health disparities: results from a ten-year, population-based study in Sweden. Stigma Health. 2018;3(1):16-26. http://dx.doi.org/10.1037/sah0000066.

38. Costa ABC, Nardi HC. O casamento "homoafetivo" e a política da sexualidade: implicações do afeto como justificativa das uniões de pessoas do mesmo sexo. Rev Estud Fem. 2015;23(1):137-50. http://dx.doi. org/10.1590/0104-026X2015v23n1p/137.

39. Grupo Gay da Bahia. Mortes violentas de LGBT+ no Brasil [Internet] 2019 [citado em 2021 ago 11]. Disponível em: https://grupogaydabahia.com.br/relatorios-anuais-de-morte-de-lgbti/

40. Mendes WG, Silva CMFP. Homicide of lesbians, gays, bisexuals, travestis, transexuals, and transgender people (LGBT) in Brazil: a spatial analysis. Cien Saude Colet. 2020;25(5):1709-22. http://dx.doi. org/10.1590/1413-81232020255.33672019. PMid:32402041.

41. Eller J, Mendes A. Autor de ação no STF que criminalizou homofobia reage à AGU: 'querem carta em branco para discriminar'. O Globo [Internet], 15 de outubro de 2020 [citado em 2021 ago 11]. Disponível em: https://oglobo.globo.com/sociedade/autor-de-acao-no-stf-que-criminalizou-homofobia-reage-aguquerem-carta-em-branco-para-discriminar-24694089

42. Rich A. Compulsory heterosexuality and lesbian existence. In: Gelp BC, Gelp A, editors. Adrienne Rich's poetry and prose. New York W.W. Norton \& Company; 1993.

43. Facchini R, Sívori H. Conservadorismo, direitos, moralidades e violência: situando um conjunto de reflexões a partir da Antropologia. Cadernos Pagu. 2017;50(50):e175000. http://dx.doi.org/10.1590/18094449201 700500000.

44. Malta RB, Flexor CLO, Costa AAN. Uma nova velha história: sobre censura e literatura LGBT+. Estud Lit Bras Contemp. 2020;61(61):e6110. http://dx.doi.org/10.1590/2316-40186110.

45. Braga DC, Bonamigo EL, Chiocca N, Munaretto BB, Moraes MM, Warmling D. Discriminação por preferência sexual nos serviços de saúde: um estudo da Pesquisa Nacional de Saúde (PNS 2013). Rev AMRIGS. 2020;64(2):231-6.

46. Barros AJ, Hirakata VN. Alternatives for logistic regression in cross-sectional studies: an empirical comparison of models that directly estimate the prevalence ratio. BMC Med Res Methodol. 2003;3(1):21. http://dx.doi.org/10.1186/1471-2288-3-21. PMid:14567763.

47. Coutinho LMS, Scazufca M, Menezes PR. Methods for estimating prevalence ratios in cross-sectional studies. Rev Saude Publica. 2008;42(6):992-8. http://dx.doi.org/10.1590/S0034-89102008000600003. PMid:19009156.

48. Boccolini CS, Boccolini PMM, Damacena GM, Ferreira APS, Szwarcwald CL. Fatores associados à discriminação percebida nos serviços de saúde do Brasil: resultados da Pesquisa Nacional de Saúde, 2013. Cien Saude Colet. 2016;21(2):371-8. http://dx.doi.org/10.1590/1413-81232015212.19412015. PMid:26910145.

49. Brasil. Lei no 8.080 de 19 de setembro de 1990. Dispõe sobre as condições para a promoção, proteção e recuperação da saúde, a organização e o funcionamento dos serviços correspondentes e dá outras providências. Diário Oficial da União [Internet], Brasília, 20 de setembro de 1990. 